\title{
A bispecific chimeric antigen receptor molecule enhances $T$ cell activation through dual immunological synapse formation and offsets antigen escape in glioblastoma
}

Meenakshi Hegde ${ }^{1 *}$, Zakaria Grada', Antonella Pignata ${ }^{1}$, Amanda Wakefield ${ }^{1}$, Kristen Fousek ${ }^{1}$, Kevin Bielamowicz ${ }^{1}$, Kevin Chow ${ }^{1}$, Vita Brawley ${ }^{1}$, Tiara Byrd ${ }^{1}$, Stephen Gottschalk¹, Malini Mukherjee ${ }^{1}$, Winfried S Wels², Matthew Baker ${ }^{1}$, Giapietro Dotti ${ }^{1}$, Jordan Orange ${ }^{1}$, Nabil Ahmed ${ }^{1}$

From 30th Annual Meeting and Associated Programs of the Society for Immunotherapy of Cancer (SITC 2015) National Harbor, MD, USA. 4-8 November 2015

\section{Background}

Antigen escape tumor cell variants prevail in tumors recurring after treatment with chimeric antigen receptor (CAR) T cells with a single specificity. Recurrent tumors preserve alternative non-targeted tumor associated antigens.

\section{Hypothesis}

A bispecific CAR will mitigate antigen escape enhancing the antitumor activity of $\mathrm{T}$ cells.

\section{Methods and results}

HER2 and IL13R $\alpha 2$ are currently targeted in Phase I glioblastoma (GBM) trials using CAR T cells. We created a bispecific CAR molecule with a HER2-specific $\mathrm{scFv}$ joined in tandem to an IL13R $\alpha 2$-binding moiety in the CAR exodomain (Tandem CAR) and a CD28. $\zeta$ signaling endodomain. We used computational modeling to interrogate this design. GBM patients' Tandem CAR $T$ cells showed distinct binding to soluble HER2 and IL13R $\alpha 2$ and killed primary autologous GBM cells. Three-dimensional reconstitution and quantification of confocal images of the Tandem CAR T cell/tumor interface revealed enhanced bifunctional immunological synapses compared to conventional CARs. Further, Tandem CAR T cells exhibited significantly enhanced inexhaustible activation dynamics when compared to conventional HER2 or IL13R 22 CAR T cells and better controlled established GBM in an orthotopic murine model by offsetting both HER2 and IL13R $\alpha 2$ escape.

\section{Conclusion}

Tandem chimeric antigen receptors enhance $\mathrm{T}$ cell activation and mitigate antigen escape through bifunctional immunological synapse formation in GBM.

\section{Authors' details}

${ }^{1}$ Baylor College of Medicine, Houston, TX, USA. ${ }^{2}$ Georg-Speyer-Haus, Institute for Tumor Biology and Experimental Therapy, Frankfurt, Germany.

Published: 4 November 2015

\section{doi:10.1186/2051-1426-3-S2-O3}

Cite this article as: Hegde et al:: A bispecific chimeric antigen receptor molecule enhances $\mathrm{T}$ cell activation through dual immunological

synapse formation and offsets antigen escape in glioblastoma. Journal for ImmunoTherapy of Cancer 2015 3(Suppl 2):03.

${ }^{1}$ Baylor College of Medicine, Houston, TX, USA

Full list of author information is available at the end of the article 


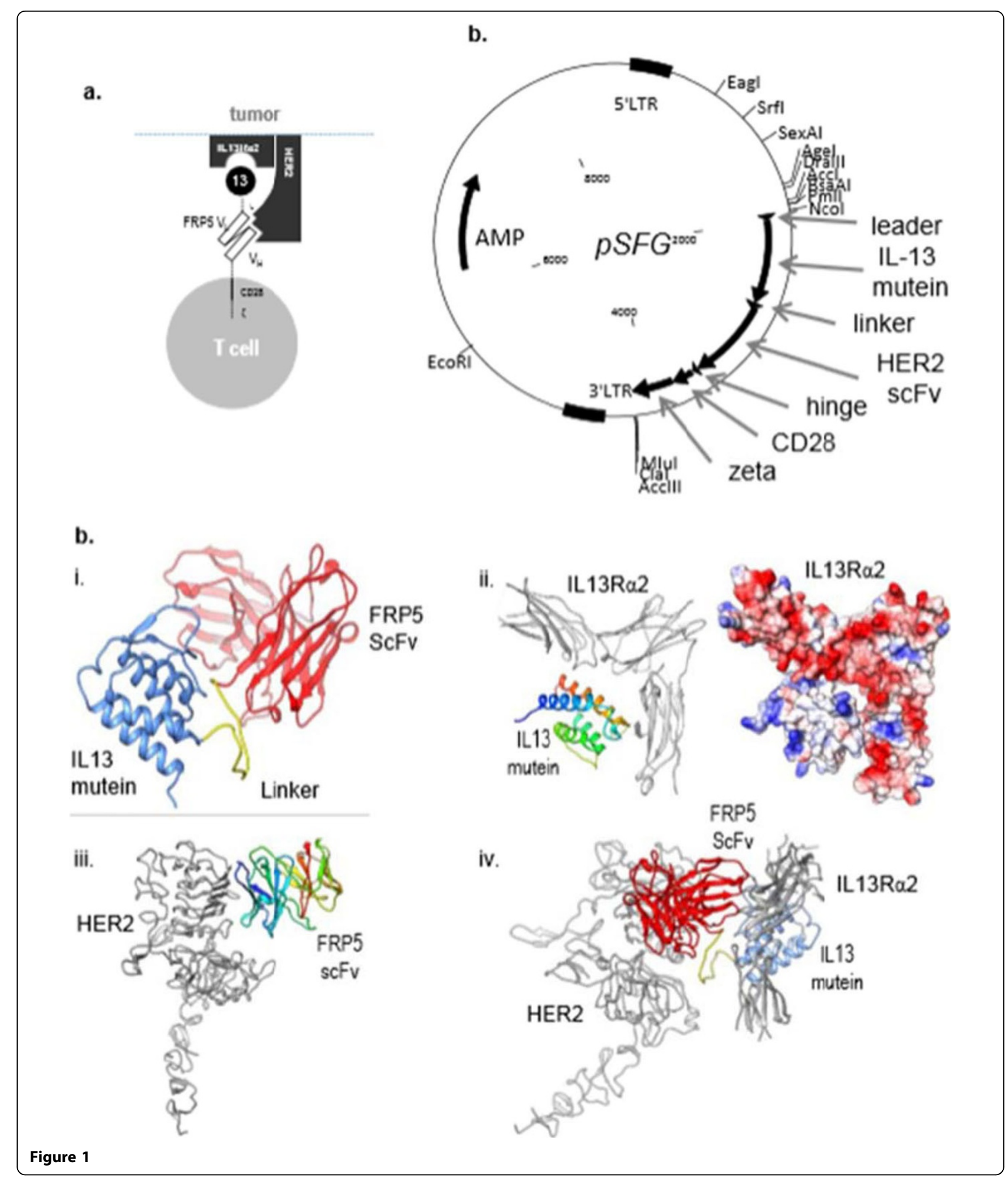



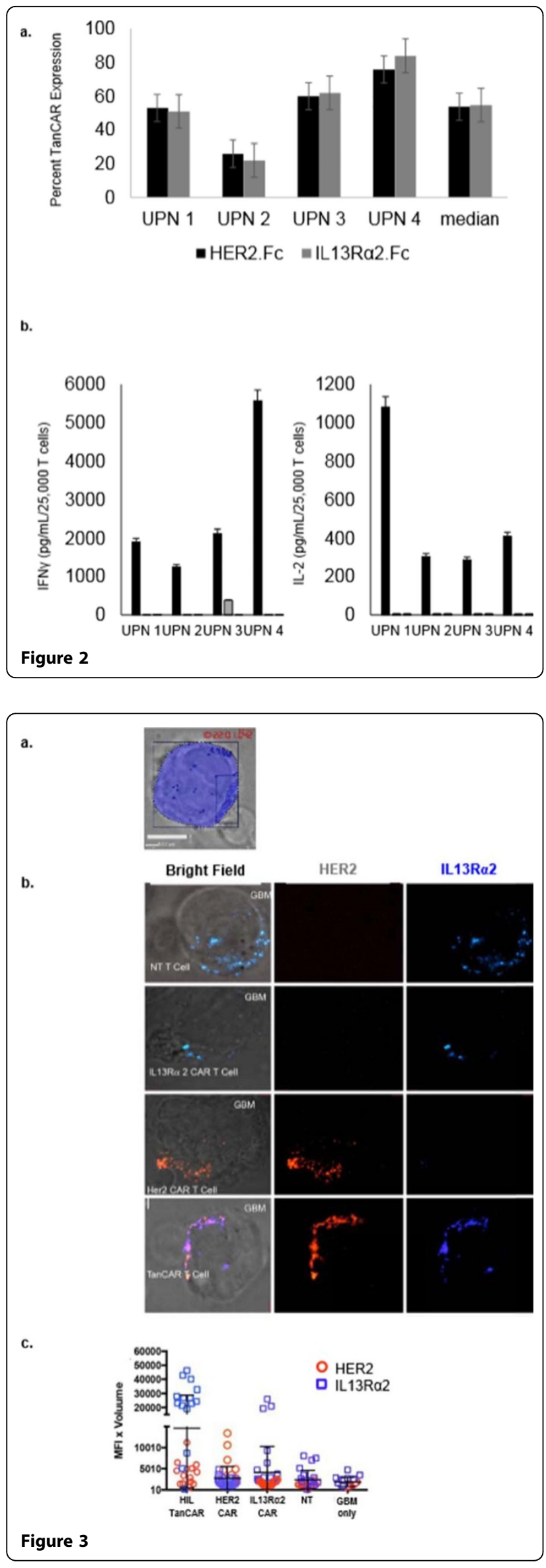
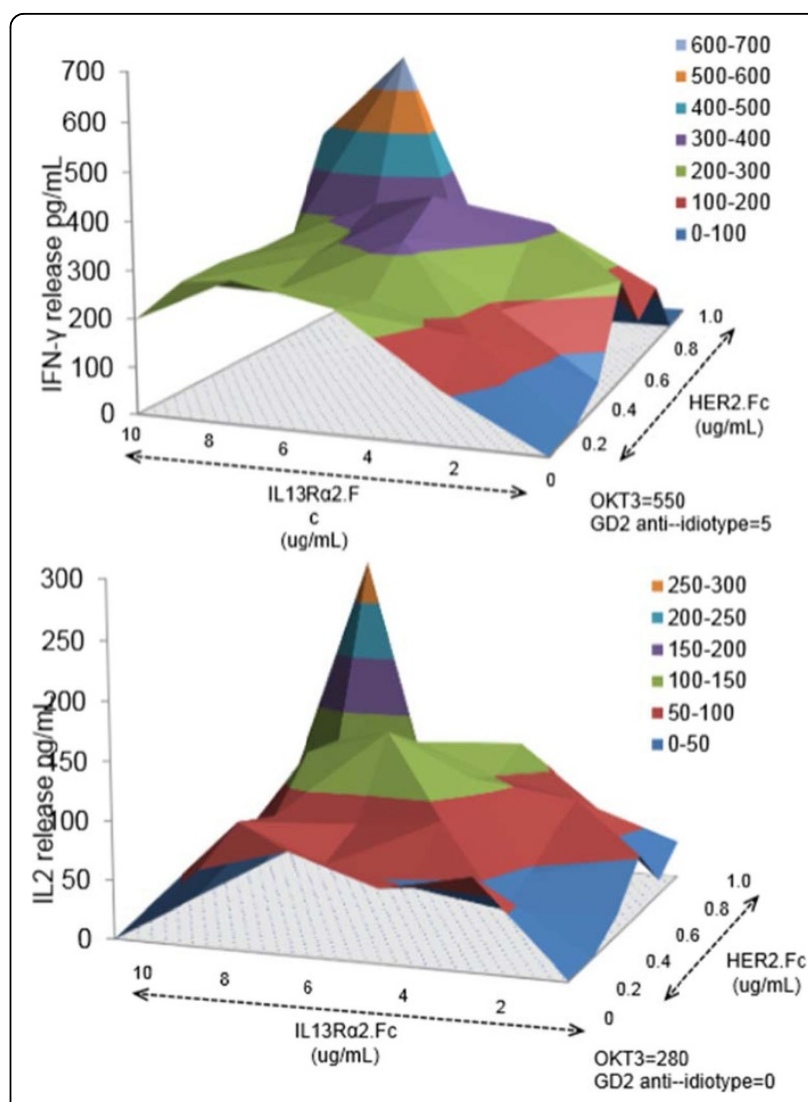

Figure 4

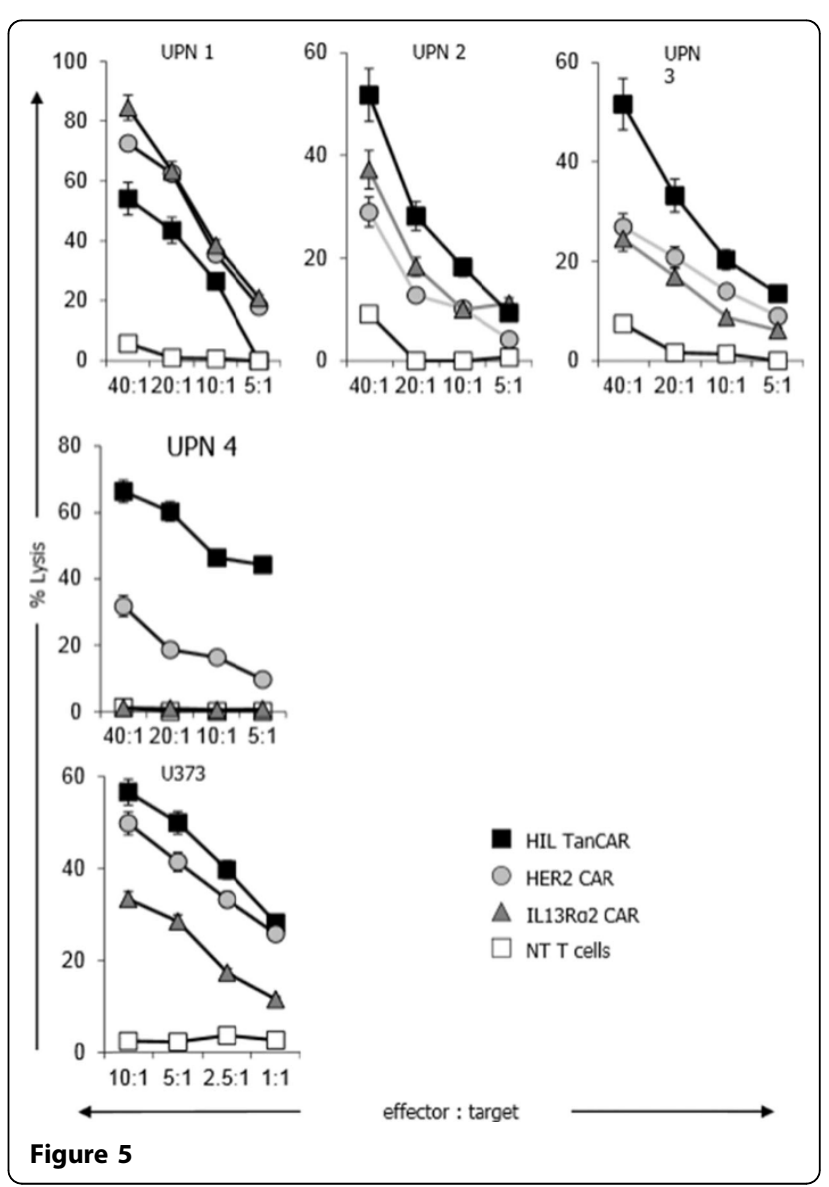



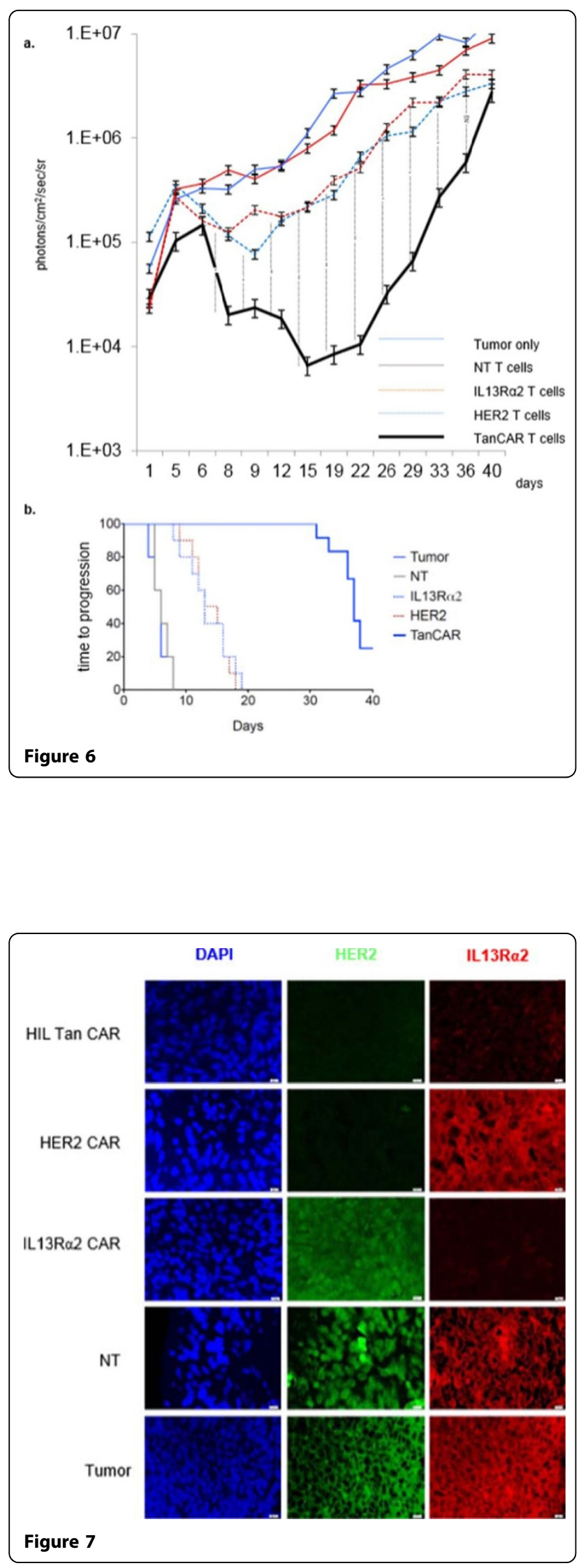